\title{
Epidemiology Characteristics of Streptococcus pneumoniae From Children With Pneumonia in Shanghai: A Retrospective Study
}

\author{
Wantong Zhao, Fen Pan, Bingjie Wang, Chun Wang, Yan Sun, Tiandong Zhang, \\ Yingying Shi and Hong Zhang*
}

Department of Clinical Laboratory, Shanghai Children's Hospital, Shanghai Jiaotong University, Shanghai, China

\section{OPEN ACCESS}

Edited by:

Ghassan M. Matar

American University of Beirut,

Lebanon

Reviewed by:

Krisztina M. Papp-Wallace, Louis Stokes Cleveland VA Medical

Center, United States

Michael L. Vasil,

University of Colorado Denver:

United States

*Correspondence: Hong Zhang

schjyk2015@126.com

Specialty section:

This article was submitted to

Clinical Microbiology,

a section of the journal

Frontiers in Cellular and Infection

Microbiology

Received: 01 May 2019

Accepted: 03 July 2019

Published: 18 July 2019

Citation:

Zhao W, Pan F, Wang B, Wang C,

Sun $Y$, Zhang $T$, Shi $Y$ and Zhang $H$

(2019) Epidemiology Characteristics of

Streptococcus pneumoniae From Children With Pneumonia in Shanghai:

A Retrospective Study.

Front. Cell. Infect. Microbiol. 9:258 doi: $10.3389 / f c i m b .2019 .00258$
Background: Streptococcus pneumoniae is the most common pathogen causing death in children under 5 years old. This retrospective surveillance aimed to analyze serotype distribution, drug resistance, virulence factors, and molecular characteristics of pneumonia isolates from children in Shanghai, China.

Methods: A total of 287 clinical pneumococcal isolates were collected from January to December in 2018 and were divided into community-acquired pneumonia (CAP) and healthcare-associated pneumonia (HAP) two groups according to where someone contracts the infection. All isolates were serotyped by multiplex sequential PCR and antimicrobial susceptibility testing was performed using E-test or disk diffusion method. The molecular epidemiology was analyzed using multilocus sequence typing and seven housekeeping genes were sequenced to identified the sequence types (STs). In addition, we investigated the presence of virulence genes via PCR.

Results: The most common serotypes were 19F, 6A, 19A, 23F, 14, and 6B, and the coverage rates of the 7-, 10- and 13-valent pneumococcal conjugate vaccines were 58.9, 58.9, and 80.5\%, respectively. More PCV13/non-PCV7 serotypes and higher rate of penicillin non-susceptible S. pneumoniae were seen in HAP. Molecular epidemiological typing showed a high level of diversity and five international antibiotic-resistant clones were found, including Taiwan ${ }^{19 F_{-}}-14$, Spain ${ }^{23 F_{-}}-1$, Spain ${ }^{6 B}-2$, Taiwan ${ }^{23 F_{-}}-15$ and Sweden ${ }^{15}$ A -25 . No significant difference was observed in the presence of virulence genes among the isolates obtained from CAP and HAP. All of the $S$. pneumoniae isolates carried IytA, ply, psaA, pavA, spxB, htrA, and clpP, and the carriage rate of nanA and piaA were 96.2 and $99.0 \%$. Conversely, cps $2 A, c b p A$, and pspA were present in $33.8-44.3 \%$ of the isolates.

Conclusions: Serotype changes and emerging multidrug-resistant international clones were found in current study. IytA, ply, psaA, pavA, spxB, htrA, and clpP may be good protein vaccine candidates. Long-term high-quality surveillance should be conducted to assess impact and effectiveness brought by vaccines, and provide a foundation for prevention strategies and vaccine policies.

Keywords: Streptococcus pneumonia, serotypes, antibiotic resistance, pneumococcal pneumonia, pneumococcal conjugate vaccine, virulence, children 


\section{INTRODUCTION}

Streptococcus pneumoniae is a frequent colonizer of the human nasopharynx with a colonization rate of $27-65 \%$ in children (Weiser et al., 2018), whilst the cause of both invasive pneumococcal disease (including bacteremia, meningitis, etc.) and non-invasive pneumococcal disease such as pneumonia and otitis media under the condition of the immunocompromised or microflora imbalance (GBD 2016 Lower Respiratory Infections, 2018; Weiser et al., 2018). It presents as a burden associated with high morbidity and mortality globally. As the global estimates reported, of all pneumococcal deaths in HIV-uninfected children in $2015,81 \%$ of them died of pneumonia (Wahl et al., 2018). Centers for Disease Control and Prevention of America recommends that pneumonia can be divided into two types according to place where someone contracts the infection, community-acquired pneumonia (CAP) which is defined as when someone develops pneumonia in the community (not in a hospital) and healthcare-associated pneumonia (HAP) which is defined as when someone develops pneumonia during or following a stay in a healthcare facility ${ }^{1}$.

In the lower respiratory infections in 195 countries in 2016, S. pneumoniae was estimated to be responsible for 341029 deaths of children younger than 5 years (GBD 2016 Lower Respiratory Infections, 2018). By far, lower respiratory infection incidence and mortality in children is mostly attributed to pneumococcal pneumonia. Vaccines and antibiotics are considered as effective methods against $S$. pneumoniae. Immunizing with vaccines was suggested by WHO to prevent $S$. pneumoniae infections (Pneumococcal vaccines WHO position paper, 2012). A reduction in CAP of $>40 \%$ after introduction of PCV7 has also been reported (Falup-Pecurariu, 2012). In Shanghai, pneumococcal vaccines belong to the second category of vaccines and vaccination is given only on an voluntary basis at their own expense, which maybe the cause of low vaccination rate of PCV. On the other hand, antimicrobial therapy is the common anti-infection treatment. However, with the changes in S. pneumoniae serotype and antibiotic resistance over time, the current treatment options are constantly being adjusted as well. The epidemiological data of pneumococcus on children with pneumonia in Shanghai is scarce at present. In this study, we aimed to analyze serotype distribution, antibiotic resistance, virulence factors and molecular characteristics of pneumonia isolates identified from children in Shanghai to provide data support for development of pneumococcal infection prevention strategies and vaccines.

\section{MATERIALS AND METHODS}

\section{Clinical Isolates and Population}

The retrospective surveillance was conducted at Shanghai Children's Hospital, which is the first specialist children's hospital in China, with about 2.5 million outpatients visiting and 44,000 hospitalized each year. A total of 287 S. pneumoniae

${ }^{1}$ Prevention CfDCa. Causes of Pneumonia. Available online at: https://www.cdc. gov/pneumonia/causes.html. isolates were collected from patients diagnosed with pneumonia between January and December in 2018. CAP included the isolates obtained from an outpatient or collected earlier than $48 \mathrm{~h}$ after hospitalization, while specimens obtained more than $48 \mathrm{~h}$ after admission were included as HAP in this investigation (Sader et al., 2018).

Clinical and epidemiological information was systematically extracted from the medical records, including demographics of the patient, symptoms and findings at hospitalization, underlying, and other potential characteristics. The protocol for present study was approved by the Shanghai Children's Hospital Ethics Committee (Shanghai Jiao Tong University School of Medicine). The retrospective study was to obtain the genus and species of the bacteria and did not affect the patients, the Review Board consequently exempted the informed consent requirements. Only one isolate was collected from each patient. Duplicate strains and patients colonized by bacteria with no clinical symptoms were excluded from the study.

\section{Microbiology Methods}

The pneumococcal isolates analyzed in current study were collected and cultured in line with the need of clinical procedures. Specimens were collected by professional staff or doctors and transported to the department of clinical microbiology within $2 \mathrm{~h}$, which were inoculated onto $5 \%$ sheep blood agar plates and incubated at $35^{\circ} \mathrm{C}, 5 \% \mathrm{CO}_{2}$ for $18-24 \mathrm{~h}$. All isolates were identified by typical colony morphology, optochin assays and confirmed by the matrix-assisted laser desorption ionizationtime of flight-mass spectrometry (MALDI-TOF MS; Bruker Daltonik GmbH, Bremen, Germany). Strains identified as $S$. pneumoniae were stored in $40 \%$ sterile glycerol broth at $-80^{\circ} \mathrm{C}$ for subsequent analysis.

\section{Serotyping}

$S$. pneumoniae isolates were serotyped by multiplex sequential PCR (MP-PCR), and a primer pair targeting $c p s A$ was used as a positive control in each reaction (Pai et al., 2006). Serogroup $6 \mathrm{~A} / \mathrm{B}$ were identified using the method described previously (Jin et al., 2009). If the serotype was not detected by the method mentioned above, the strain was classified as non-typeable. Afterwards, the coverage rates of PCV7, PCV10, and PCV13 were estimated by calculating the percentage of isolates expressed the serotypes included in the vaccines.

\section{In vitro Antimicrobial Susceptibility Testing}

Antimicrobial resistance testing of all 287 isolates were determined by E-test and Kirby-Bauer disk tests. In our study, we used E-test assay (AB Biodisk, Solna, Sweden) to measure the minimum inhibitory concentrations (MICs) to penicillin. The susceptibility to clindamycin, erythromycin, linezolid, moxifloxacin, sulfamethoxazole-trimethoprim and vancomycin was assessed using the disk diffusion method (Oxoid Ltd, Basingstoke, UK). All susceptibility tests and results interpretations were performed following the guidelines and criteria established by the Clinical and Laboratory Standard Institute (CLSI) 2018. The quality-control strain was $S$. pneumoniae ATCC 49619, which included in each set of tests 
to ensure the reliability of the results. Isolates resistant to three or more kinds of antibiotics tested were defined as MDR $S$. pneumoniae in this study.

\section{Multilocus Sequence Typing}

To determine the STs of the isolates, multilocus sequence typing (MLST) analysis was carried out in accordance with the $S$. pneumoniae MLST protocol (Enright and Spratt, 1998). In our experiment, we used the seven housekeeping genes (aroE, $g d h, g k i$, recP, spi, xpt, and $d d l$ ), which were amplified by PCR using primers previously described (Enright and Spratt, 1998). The internal fragments amplified were sequenced on both strands by the Sanger method using the primers that were used for the initial amplification. Alleles and sequence types (STs) were confirmed by querying the pneumococcal MLST database (http://pubmlst.org/spneumoniae/). The STs obtained were then compared with Pneumococcal Molecular Epidemiology Network (PMEN) clones (http://www.pneumogen.net/pmen/). STs that were different from any known ST were submitted for new name assignment. The relatedness between the isolates was constructed by eBURST version 3.0 software. Strains were assigned to a clonal complex (CC) based on the stringent group definition of six of seven shared alleles (Feil et al., 2004).

\section{Detection of Virulence Genes}

A total of 12 genes related to virulence were detected by PCR using published primers (Ibrahim et al., 2004; Shakrin et al., 2014; Bryant et al., 2016; Kang et al., 2016), including capsular polysaccharide (cps2A), autolysin (lytA), pneumococcal surface protein A $(p s p A)$, choline binding protein $\mathrm{A}(c b p A)$, neuraminidase (nanA), ion transporters (piaA), pneumolysin (ply), pneumococcal surface adhesin A (psaA), pneumococcal adherence and virulence factor A ( $p a v A)$, pyruvate oxidase $(s p \times B)$, serine protease high-temperature requirement $\mathrm{A}(h t r A)$, and caseinolytic protease $(c l p P)$. The PCR products were analyzed by gel electrophoresis and sequencing. The positive products were confirmed by comparing to the online database via BLAST.

\section{Statistical Analysis}

Antibiotic resistance was analyzed with the WHONET 5.6 software, while SPSS 24.0 was used for statistical analysis. Chisquare test or Fisher's exact test were used for significance comparison of categorical data, whereas $t$-test or Rank-sum test were used for comparing quantitative data. $\mathrm{P}<0.05$ was considered to be statistically significant.

\section{RESULTS}

\section{Demographic and Clinical Characteristics}

The total collection presented 287 S. pneumoniae isolates causing pneumonia, of which 243 from CAP and 44 from HAP. As was shown in Table 1, 90.9\% of these strains (261/287) were isolated from children aged $0-5$ years old and the male to female sex ratio was 1.3:1. Number of cases diagnosed in summer (during June to August) were a little lower than other seasons. The common chronic diseases in this study are congenital heart disease
(28/287) and asthma (14/287). Concurrent infection was noted in $48.8 \%$ patients. No children were vaccinated with pneumococcal conjugate vaccine $(\mathrm{PCV})$ in current study. In the aggregate, discharge data showed that all patients had a favorable prognosis. CAP was the most common in the respiratory department whereas the rate of HAP in the gastroenterology dept is higher. There is no statistical difference in the sex, age, season, prognosis and other clinical and demographic characteristics between CAP and HAP.

\section{Serotype Distribution and Vaccine Coverage}

Of the 287 pneumococcal isolates, 261 isolates (90.9\%) were successfully serotyped and $19 \mathrm{~F}(33.4 \%)$ was the most common serotype, followed by $6 \mathrm{~A}(11.8 \%), 19 \mathrm{~A}(9.8 \%), 23 \mathrm{~F}(8.4 \%), 14$ (8.4\%), 6B (8.0\%), $34(2.8 \%), 15 \mathrm{~B} / \mathrm{C}(2.4 \%)$, and $15 \mathrm{~A}(2.1 \%)$. Other uncommon serotypes were detected in fewer than five strains each, which included serotype 7C (3), 11A (3), 20 (1), 4 (1), 33F (1), 9V (1), and 18 (1). The rest 26 isolates were classified as non-typeable. The serotype distribution of pneumococcal strains isolated is shown in Figure 1.

The overall vaccine coverage rates of PCV7, PCV10, and PCV13 serotypes were 58.9, 58.9, and 80.5\%, respectively. HAP had a lower vaccine serotype coverage rate than CAP. A higher rate of PCV13/non-PCV7 serotypes was noticed in HAP. Simultaneously, serotype 4, 9V, 15B/C, 7C, 18, 20 and 33F were only observed in CAP.

\section{Antibiotic Susceptibility}

The total prevalence of penicillin non-susceptible $S$. pneumoniae (PNSP) was $31.7 \%$ including penicillin-intermediate $S$. pneumoniae (PISP, 26.8\%) and penicillin-resistant $S$. pneumoniae (PRSP, 4.9\%) (Table 2). Most strains showed high resistance to erythromycin and clindamycin (>95\%). No drug-resistant strains to linezolid, moxifloxacin and vancomycin were observed in this study. In addition, resistance to sulfamethoxazole-trimethoprim was seen in $76.7 \%$ of isolates.

Approximately $74.9 \%$ (215/287) of the isolates were defined as MDR. Resistance of pneumococcus to the agents above among different serotypes was also assessed and it was found that the antibiotic resistance varied by serotype. Serotypes 19F, $19 \mathrm{~A}$, and $23 \mathrm{~F}$ prevailed in PNSP isolates, and almost all 19F isolates were resistant to sulfamethoxazole-trimethoprim. PCV13 covered $85.1 \%(183 / 215)$ of the MDR strains, which was higher than that for PCV7 $(61.4 \%, 132 / 215)$. Emerging serotypes (11A, $15 \mathrm{~B} / \mathrm{C}, 18,20,34,7 \mathrm{C}$, and $9 \mathrm{~A}$ ) accounted for $8.4 \% \mathrm{MDR}$. Compared with CAP, there were higher rates of PNSP (30.9 vs. $36.4 \%)$ and MDR (74.5\% vs. $77.3 \%)$ in HAP.

\section{MLST}

Sixty-four STs were identified by MLST analysis among the 287 isolates. The five predominant STs were ST271 $(n=72$, $25.1 \%)$, ST320 ( $n=30,10.5 \%)$, ST3173 ( $n=24,8.4 \%)$, ST876 $(n=14,4.9 \%)$, and ST81 $(n=13,4.5 \%)$, which were mainly related to serotype 19F, 19A, 6A/B, 14, and 23F, respectively. Nine clonal complexes and 39 singletons were obtained using eBURST version3.0 software analysis for the homology relationship 
TABLE 1 | Clinical and demographic characteristics of children with CAP $(n=243)$ and HAP $(n=44)$ in Shanghai.

\begin{tabular}{|c|c|c|c|c|c|}
\hline & $\operatorname{CAP}(n=243)$ & HAP $(n=44)$ & Total $(n=287)$ & $\%$ & $P$ \\
\hline Sex & & & & & 0.202 \\
\hline Male & $135(55.6)$ & $29(65.9)$ & 164 & 57.1 & \\
\hline Female & $108(44.4)$ & $15(34.1)$ & 123 & 42.9 & \\
\hline Age (months) & & & & & 0.881 \\
\hline$<12$ & $49(20.2)$ & $11(25.0)$ & 60 & 20.9 & \\
\hline $12-24$ & $66(27.2)$ & $12(27.3)$ & 78 & 27.2 & \\
\hline $24-60$ & $105(43.2)$ & $18(40.9)$ & 123 & 42.9 & \\
\hline$>60$ & $23(9.5)$ & $3(6.8)$ & 26 & 9.1 & \\
\hline Disease-onset season & & & & & 0.992 \\
\hline December-February & $59(24.3)$ & $11(25.0)$ & 70 & 24.4 & \\
\hline March-May & $69(28.4)$ & $13(29.5)$ & 82 & 28.6 & \\
\hline June-August & $49(20.1)$ & $8(18.2)$ & 57 & 19.9 & \\
\hline September-November & $66(27.2)$ & $12(27.3)$ & 78 & 27.2 & \\
\hline \multicolumn{6}{|l|}{ Chronic disease } \\
\hline Congenital heart disease & $20(8.2)$ & 8 (18.2) & 28 & 9.8 & 0.077 \\
\hline Asthma & $14(5.8)$ & 0 & 14 & 4.9 & 0.211 \\
\hline \multicolumn{6}{|l|}{ Concurrent infection } \\
\hline Mycoplasma pneumoniae & $61(25.1)$ & 9 (20.5) & 70 & 24.4 & 0.509 \\
\hline Rhinovirus & $23(9.5)$ & $2(4.5)$ & 25 & 8.7 & 0.439 \\
\hline Respiratory syncytial virus & $14(5.8)$ & $2(4.5)$ & 16 & 5.6 & 1.000 \\
\hline Influenza virus & $15(6.2)$ & 0 & 15 & 5.2 & 0.185 \\
\hline Epstein-Barr virus & $8(3.3)$ & $1(2.3)$ & 9 & 3.1 & 1.000 \\
\hline Cytomegalovirus & $5(2.1)$ & 0 & 5 & 1.7 & 1.000 \\
\hline \multicolumn{6}{|l|}{ Wards of hospitalization } \\
\hline Respiratory medicine & $181(74.5)$ & $25(56.8)$ & 206 & 71.7 & 0.017 \\
\hline Priority ward & $42(17.3)$ & 9 (20.5) & 51 & 17.8 & 0.613 \\
\hline Gastroenterology & $5(2.1)$ & $5(11.4)$ & 10 & 3.5 & 0.008 \\
\hline ICU & $5(2.1)$ & $2(4.5)$ & 7 & 2.4 & 0.650 \\
\hline Others* & $10(4.1)$ & $3(6.8)$ & 13 & 4.5 & 0.690 \\
\hline Fever (days), median (IQR) & $2(1-4)$ & $2(1-4)$ & - & - & 0.657 \\
\hline Antibiotic (days), median (IQR) & $7.21(6-8)$ & $6.69(5-7.5)$ & - & - & 0.395 \\
\hline Hospitalization (days), median (IQR) & $7(6-8)$ & $7(6-9)$ & - & - & 0.112 \\
\hline Cure & $243(100)$ & $44(100)$ & 287 & 100 & \\
\hline
\end{tabular}

*Other wards of hospitalization, including Neurology, Cardiology and Neonatology, Otolaryngology-Head and Neck Surgery.

between these STs (Figure 2). Among the 9 CCs, the most prevalent clonal complex CC271 (including ST271, ST236, ST320, ST1968 etc.) accounted for $42.9 \%$ (123/287) of the isolates, followed by CC3173 (10.5\%, 30/287) and CC81 (4.9\%, 14/287).

Comparing the isolates with the PMEN clones (at least 6 of 7 MLST alleles shared), five international antibioticresistant clones were found in this study, including Taiwan ${ }^{19 F_{-}}$ 14, Spain ${ }^{23 \mathrm{~F}}-1$, Spain ${ }^{6 \mathrm{~B}}-2$, Taiwan ${ }^{23 \mathrm{~F}}-15$ and Sweden ${ }^{15 \mathrm{~A}}-25$. The isolates belonging to these international clones or their single locus variants (SLVs) made up of $40.8 \%$ of all strains. The dominating of the five international clones was Taiwan ${ }^{19} \mathrm{~F}_{-} 14$. Furthermore, CC271 related to the Taiwan ${ }^{19 F}-14$ was mainly associated with the serotype 19 group, 88 of which were serotype $19 \mathrm{~F}$ and 25 were $19 \mathrm{~A}$. Sweden ${ }^{15 \mathrm{~A}}-25$ was also identified in this study and this group of isolates included two STs, ST63 $(n=2)$ and SLV ST2248 $(n=4)$, with serotypes $15 \mathrm{~A}$ and 14 , respectively. What's more, one new aroE allele and five new STs were found in our study. There were no significant differences in the major STs and distribution between HAP and CAP.

\section{Presence and Distribution of Virulence Genes}

The presence of virulence genes did not show any significant difference among the isolates obtained from CAP and HAP (Table 3). Irrespective of the source of the isolation, all isolates carried lytA, ply, psaA, pavA, spxB, htrA, and $c l p P$ genes. In general, most of the isolates harbored nanA (96.2\%) and piaA (99.0\%). Significant association was suggested between carriage rate and serotype in $c p s 2 A, \operatorname{cbp} A, p s p A$ and nan $A$. Besides, $c p s 2 A$, $\operatorname{cbp} A$, and $p s p A$ was also associated with clonal complex.

cps $2 \mathrm{~A}$ was present in $44.3 \%$ isolates and all serotypes $19 \mathrm{~A}$ and 14 possessed it. The majority of serogroup 19 isolates carried $c b p A$, including $19 \mathrm{~F}(86.5 \%)$ and $19 \mathrm{~A}$ (89.3\%). Serotypes $6 \mathrm{~A}$ and $6 \mathrm{~B}$ were the most dominant serotypes to carry $p s p A$. 


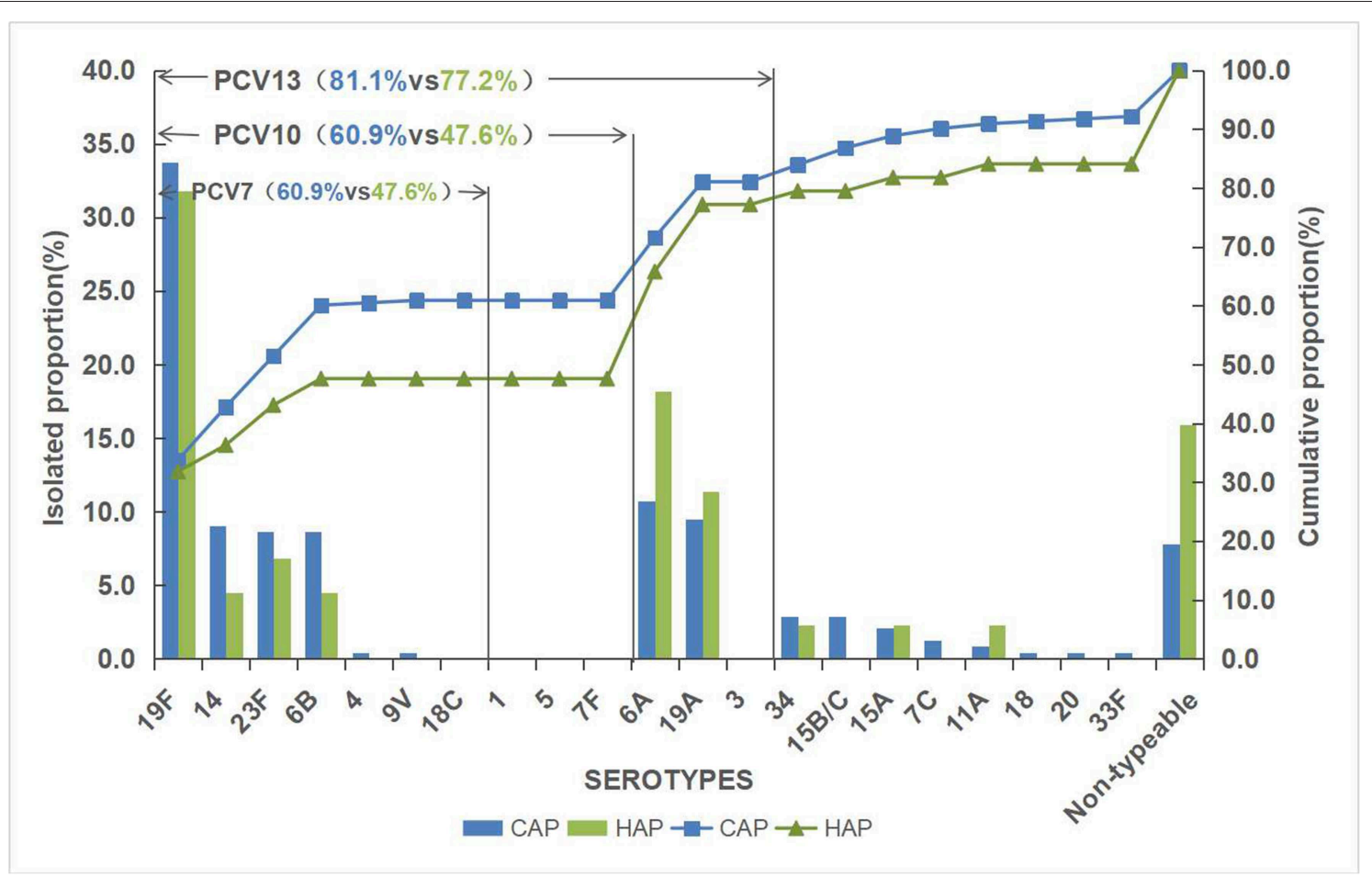

FIGURE 1 | Serotype distribution of pneumococcal strains isolated in Shanghai in 2018.

TABLE 2 | Antimicrobial resistance of pneumococcal isolates from children with CAP $(n=243)$ and HAP $(n=44)$.

\begin{tabular}{|c|c|c|c|c|c|c|c|c|c|}
\hline Agent (\%) & \multicolumn{3}{|c|}{ CAP $(N=243)$} & \multicolumn{3}{|c|}{ HAP $(N=44)$} & \multicolumn{3}{|c|}{ TOTAL $(N=287)$} \\
\hline SXT & 69.0 & 92.3 & 90.0 & 67.9 & 100 & 100 & 68.9 & 93.5 & 92.9 \\
\hline ERY & 99.4 & 96.9 & 100 & 100 & 100 & 100 & 99.5 & 97.4 & 100 \\
\hline CLI & 98.8 & 96.9 & 80.0 & 100 & 100 & 75.0 & 99 & 97.4 & 78.6 \\
\hline MXF & 0 & 0 & 0 & 0 & 0 & 0 & 0 & 0 & 0 \\
\hline
\end{tabular}

SXT, sulfamethoxazole-trimethoprim; ERY, erythromycin; CLI, clindamycin; VAN, vancomycin; LZD, linezolid; MXF, moxifloxacin.

The relationship between virulence patterns and serotypes of S. pneumoniae isolated from CAP and HAP was listed in Table 4. Based on the studied genes, the most common virulence pattern in current study was lytA-ply-psaA-pavA-spxB-htrA-clpPcbpA-nanA-piaA (27.9\%), with $19 \mathrm{~F}$ accounting for the majority and $90 \%$ were MDR, followed by pattern lytA-ply-psaA-pavA$s p x B-h t r A-c l p P-c p s 2 A-n a n A-p i a A(16.7 \%)$ that contains a variety of serotypes.

\section{DISCUSSION}

WHO reported that pneumonia accounts for $16 \%$ of all deaths of children under 5 years old, killing 920,136 children in 2015, with the most common cause of bacterial pneumonia being $S$. pneumoniae $^{2}$. In this study, we identified a total of 287 pediatric patients diagnosed with pneumococcal pneumonia from January to December 2018 in Shanghai, China. Most of them (261,90.9\%) were under 5 years old and diagnosed less in the summer, which was in line with the recent results from China (Cai et al., 2018). A male sexual superiority was also noticed among the study population, so as in other studies (Cai et al., 2018; Arushothy et al., 2019). In current study, chronic diseases were observed in $15.7 \%$ patients, which is an independent risk factor for pneumonia-related mortality in children (Zhang et al., 2013; Sonego et al., 2015; Nguyen et al., 2017). HAP has a higher

\footnotetext{
${ }^{2}$ World Health Organization. Pneumonia-Key facts. Available online from: https:// www.who.int/news-room/fact-sheets/detail/pneumonia.
} 




rate of congenital heart disease than CAP, and more CAP with asthma. Meanwhile, we also noted almost half of children were coinfected with Mycoplasma pneumoniae or Respiratory syncytial virus, etc. Possibly because viral respiratory tract infections are a major facilitator of pneumococcal infections (Smith et al., 2014; Cawcutt and Kalil, 2017).

Our present study demonstrated that the most common serotypes among children in Shanghai were 19F, $6 \mathrm{~A}, 19 \mathrm{~A}, 23 \mathrm{~F}, 14,6 \mathrm{~B}$, and 34 and the serotype coverage of PCV 13 was $80.5 \%$, which was similar to other recent studies in China but the ranking orders varied (Li et al., 2018; Shi et al., 2019). However, these serotypes were different from those in Latin America and the Caribbean (Gentile et al., 2012). PCV13 covered more isolates in Shanghai than in other countries (Miyazaki et al., 2017; Dalcin et al., 2018), probably because it was just licensed for optional use in 2016 and had not been taken into the standard childhood immunization program in mainland China. Compared with our previous reports (Pan et al., 2015a,b), the proportion of serotype $19 \mathrm{~A}$ and $23 \mathrm{~F}$ decreased while 14 and 34 increased. There was a reduction of $2-5 \%$ in serotype $15 B / C$, replaced by the appearance of $15 \mathrm{~A}$, mainly from CAP. As the methods for serotype in this study have technical limitations, 26 strains were identified as non-typeable, and most of them were from HAP. The PCV 13 coverage was a little lower than that prior to it was licensed in China, which may be on account of the selection of vaccines or natural fluctuations. Although the vaccine is not widely used, we still observed the phenomenon of serotype changes, which suggests that the changes in serotype maybe not directly related to vaccination. Studies indicated that pneumococci was able to change their capsular serotype by exchanging the capsular locus genes (Coffey et al., 1998). In our study, the serotype coverage of PCV13 remains much higher than the average rate of other regions in China (68.4\%) (Chen et al., 2018). Hence, vaccination is of great importance to eliminate the burden of pneumococcal infection in Shanghai. Nevertheless, other studies discussed serotypes rates within the vaccine will decrease by $50 \%$ due to PCV13, which becomes a problem (Shiri et al., 2017; Suzuki et al., 2017). There were rapid and substantial reductions of disease caused by PCV-serotypes (children aged $<5$ years old) in Australia, Canada, England and Wales, South Africa and the USA after the introduction of pneumococcal vaccines, subsequently an increase in the incidence of diseases caused by non-PCV7 serotypes (Pneumococcal vaccines WHO position paper, 2012). And that the distribution of serotypes vary across different affected populations as well as economic development, and change over time (Johnson et al., 2010; Zhao et al., 2017; Yan et al., 2019), so these vaccines should be reevaluated systematically and monitored long-term.

Penicillin represents as the first choice for the antibiotic treatment of S. pneumoniae infections, but the resistance to it has continued to increase across the world (Linares et al., 2010). Its susceptibility rates varied from $70.7 \%$ in Europe to $52.4 \%$ in Asia-Pacific region for all years combined from 1997 to 2016 (Sader et al., 2019). In comparison with previous study (Pan et al., 2015b), there is a significant increase in the proportion of PNSP (from 20 to $31.7 \%$ ), which means approximately one third of the isolates in this study were non-susceptible to penicillin. In addition, decrease in erythromycin susceptibility was observed as well, and compared with North America whose susceptibility rate was $55.0-56.0 \%$, its resistance is very severe in our study 


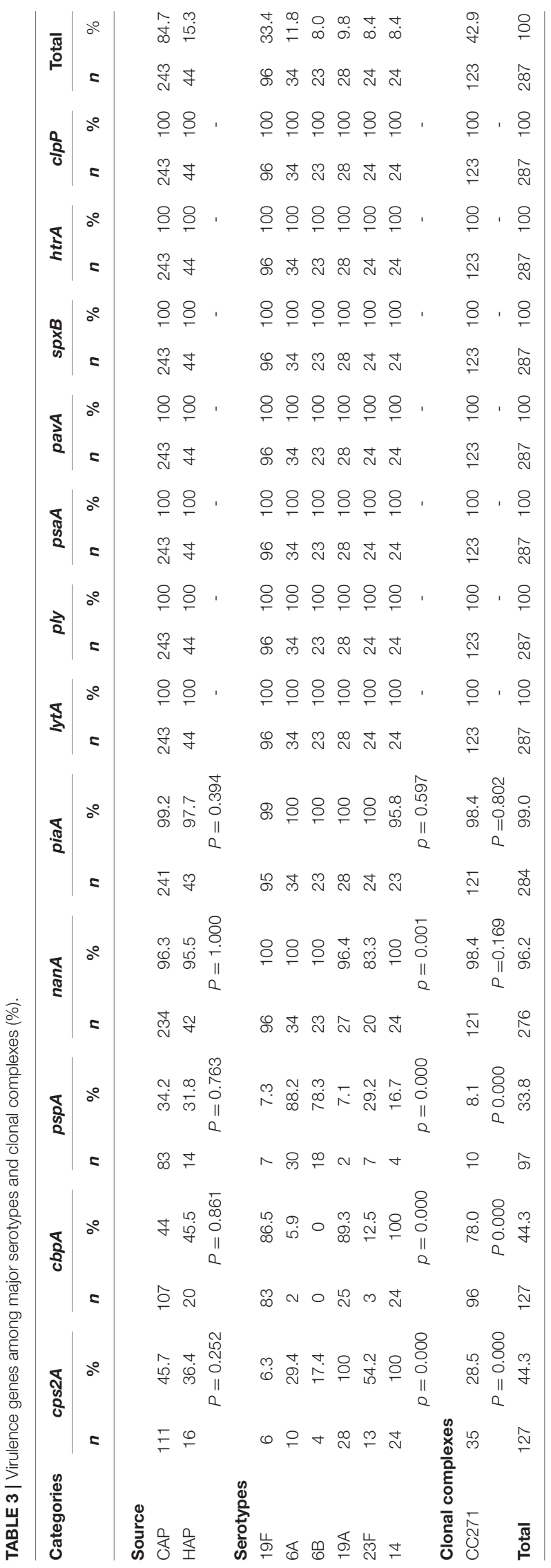

(Sader et al., 2019). It was reported that the susceptibility rates were lower among isolates from pediatric patients than adults for penicillin and azithromycin (Sader et al., 2019). In the Chinese consensus, antibiotics use in children is restricted due to the concern for safety and risk of adverse events. For instance, despite the widely use of fluoroquinolones for their highly effective in adults, their use in children is limited because of the side-effect and toxicity they may cause, such as destructive arthropathy and influence on the central nervous system (Patel and Goldman, 2016). The increase resistance maybe attributed to the selection pressure caused by the widespread use of $\beta$ lactam and macrolide antibiotics that were used as first-line therapy in children for their little side-effect and lower toxicity (Bradley et al., 2011). HAP with a higher rate of PNSP and proportion of MDR than CAP was noticed in this study, which suggested that the strains within the community were more susceptible and the strains obtained within hospital were more resistant. The higher resistance in HAP may be due to the higher rates of PCV13/non-PCV7 serotypes because of the significant relationship between antibiotic resistance and serotypes. Our data showed that PCV13 covered $85.1 \%$ MDR isolates, which suggested vaccine has the potential to control the spread of MDR (Maraki et al., 2010, 2018). Against all isolates vancomycin, linezolid and moxifloxacin exhibited good activity, which could be alternatives for treatment of PRSP and MDRSP infections.

The most common STs in this study was ST271,ST320,ST3173, and ST876, which was similar to other reports in other regions of China (Li et al., 2018; Shi et al., 2019; Yan et al., 2019). When comparing with the multi-center study in Shanghai in 2013 (Pan et al., 2015b), we observed that the dominating STs were basically the same, with a reduction in ST81 and increase in ST3173 and ST876. The rate of clones registered as MDR PMEN clones was essentially equal but different in content composition. Except for four common international antibiotic-resistant clones Taiwan ${ }^{19 F_{-}}$-14, Spain ${ }^{23 F_{-}}$, Spain ${ }^{6 \mathrm{~B}}$ 2 and Taiwan ${ }^{23 \mathrm{~F}}-15$ in Shanghai, Sweden ${ }^{15 \mathrm{~A}}-25$ was newly observed in our study. After the introduction of PCV13, the increasing serotype $15 \mathrm{~A}$ was noted in Norway, Canada and Japan (Steens et al., 2013; Ricketson et al., 2014; Naito et al., 2016). In our study, six isolates belongs to Sweden ${ }^{15 \mathrm{~A}}-25 / \mathrm{ST} 63$ and its single locus variant. Most of the clones in 2018 carried the same serotypes in 2013. In addition, ST2248, a SLV of Sweden ${ }^{15 \mathrm{~A}}-25 / \mathrm{ST} 63$, were detected in serotype 14. Five new STs were also obtained, related to serotype $34,6 \mathrm{~A} / \mathrm{B}$ and $15 \mathrm{~B} / \mathrm{C}$. With the popularization of vaccines, a careful monitoring should be required in the future about the Sweden ${ }^{15 \mathrm{~A}}-25 / \mathrm{ST} 63$ known as one of the MDR PMEN clones (Hackel et al., 2013).

Dispensable genes, not required for bacterial growth, provide survival advantages to $S$. pneumoniae. We detected the prevalence of a total of 12 virulence factors of particular important. These genes play a role in adherence to host cells, evasion of host immune responses and promotion of the biofilms formation to enhance bacterial survival competitiveness (Brooks and Mias, 2018). Comparison with other investigations, the detection rate varied across the regions and isolates disease related or colonization-related (Qin et al., 2013; Fu et al., 2019). Our data indicated that there is no difference between 
TABLE 4 | The relationship between virulence pattern and serotypes of $S$. pneumoniae isolated from CAP and HAP.

\begin{tabular}{|c|c|c|c|c|c|}
\hline Virulence pattern & $\begin{array}{l}\text { Isolates } \\
\text { (No.) }\end{array}$ & $\begin{array}{l}\text { Proportion } \\
\text { (\%) }\end{array}$ & $\begin{array}{l}\text { CAP } \\
\text { (No.) }\end{array}$ & $\begin{array}{l}\text { HAP } \\
\text { (No.) }\end{array}$ & Related serotypes (No.) \\
\hline lytA-ply-psaA-pavA-spxB-htrA-clpP-pspA & 1 & 0.3 & 0 & 1 & untyped (1) \\
\hline lytA-ply-psaA-pavA-spxB-htrA-clpP-piaA & 1 & 0.3 & 1 & 0 & Untyped (1) \\
\hline lytA-ply-psaA-pavA-spxB-htrA-clpP-cps2A-nanA & 1 & 0.3 & 1 & 0 & $14(1)$ \\
\hline IytA-ply-psaA-pavA-spxB-htrA-clpP-cps2A-piaA & 1 & 0.3 & 1 & 0 & $33 F(1)$ \\
\hline IytA-ply-psaA-pavA-spxB-htrA-clpP-pspA-piaA & 2 & 0.7 & 2 & 0 & $23 F(2)$ \\
\hline lytA-ply-psaA-pavA-spxB-htrA-clpP-nanA-piaA & 29 & 10.1 & 22 & 7 & 19F (8),6A (3),6B (4),23F (5),15A (1),18 (1),untyped (7) \\
\hline lytA-ply-psaA-pavA-spxB-htrA-clpP-cbpA-nanA & 1 & 0.3 & 1 & 0 & $19 F(1)$ \\
\hline lytA-ply-psaA-pavA-spxB-htrA-clpP-cps2A-pspA-piaA & 3 & 1.0 & 3 & 0 & 19A (1),untyped (2) \\
\hline lytA-ply-psaA-pavA-spxB-htrA-clpP-cps2A-nanA-piaA & 48 & 16.7 & 44 & 4 & $\begin{array}{l}14(19), 23 \mathrm{~F}(11), 11 \mathrm{~A}(1), 15 \mathrm{~A}(1), 15 \mathrm{~B} / \mathrm{C}(4), 19 \mathrm{~A}(2), 19 \mathrm{~F} \\
(3), 20(1), 6 \mathrm{~B}(1), 9 \mathrm{~V}(1), \text { untyped (4) }\end{array}$ \\
\hline lytA-ply-psaA-pavA-spxB-htrA-clpP-cbpA-nanA-piaA & 80 & 27.9 & 67 & 13 & 19F (76),15A (2),6A (1),untyped (1) \\
\hline IytA-ply-psaA-pavA-spxB-htrA-clpP-cbpA-pspA-piaA & 1 & 0.3 & 1 & 0 & $23 F(1)$ \\
\hline lytA-ply-psaA-pavA-spxB-htrA-clpP-pspA-nanA-piaA & 38 & 13.2 & 31 & 7 & $6 \mathrm{~A}(19), 6 \mathrm{~B}(15), 23 \mathrm{~F}(3), 19 \mathrm{~F}(1)$ \\
\hline lytA-ply-psaA-pavA-spxB-htrA-clpP-cps2A-cbpA-pspA-piaA & 2 & 0.7 & 1 & 1 & 23F (1),untyped (1) \\
\hline IytA-ply-psaA-pavA-spxB-htrA-clpP-cps2A-cbpA-nanA-piaA & 29 & 10.1 & 23 & 6 & 19A (24), 19F (1),23F (1),15B/C (1),11A (1), untyped (1) \\
\hline IytA-ply-psaA-pavA-spxB-htrA-clpP-cps2A-pspA-nanA-piaA & 36 & 12.5 & 31 & 5 & $\begin{array}{l}6 \mathrm{~A}(10), 34(6), 14(4), 6 \mathrm{~B}(3), 7 \mathrm{C}(3), 19 \mathrm{~F}(1), 15 \mathrm{~B} / \mathrm{C}(1) \\
11 \mathrm{~A}(1), 4(1), \text { untyped (6) }\end{array}$ \\
\hline IytA-ply-psaA-pavA-spxB-htrA-clpP-cbpA-pspA-nanA-piaA & 7 & 2.4 & 7 & 0 & $19 \mathrm{~F}(4), 15 \mathrm{~A}(2), 6 \mathrm{~A}(1)$ \\
\hline IytA-ply-psaA-pavA-spxB-htrA-clpP-cps2A-cbpA-pspA-nanA-piaA & 7 & 2.4 & 7 & 0 & 19F (1),19A (1),34 (2),15B/C (1),untyped (2) \\
\hline
\end{tabular}

CAP and HAP isolates in the presence and distribution of virulence genes. The positive rate of $\operatorname{cps} 2 A, \operatorname{cbp} A, p \operatorname{sp} A$, and nan $A$ varied from different serotypes. It's worth noting that nine isolates were negative for the housekeeping gene $c p s A$ similar to other reports. Studies indicated that non-encapsulated $S$. pneumoniae could also cause 3-19\% of pneumococcal diseases and the current conjugate vaccines may be ineffective against them because of serotype specificity (Keller et al., 2016). The appearance of serotype replacement and non-encapsulated strains suggested a new type vaccine should be designed to prevent pneumococcal infections. Virulence genes are crucial candidate targets for the development of next-generation protein vaccines. For instance, a study reported that generate neutralizing antibodies immunization with pneumococcal neuraminidases $n a n A, n a n B$, and nanC was able to increase survival in mice (Janapatla et al., 2018). Our results showed that there is a high prevalence in nanA, piaA, lytA, ply, psaA, pavA, spxB, htrA and $c l p P$ whereas lower in $c p s 2 A, \operatorname{cbp} A$, and $p s p A$. A requisite for a vaccine candidate is that the selected gene (s) is widely distributed in the target pneumococcal population (Cornick et al., 2017). The extremely high carriage rate suggests the potential to develop vaccines. However, this current study suggests that $\operatorname{cps} 2 A, \operatorname{cbp} A$, and $p s p A$ might be not suitable to be candidates for vaccines in Shanghai.

In conclusion, our study described the epidemiology characteristics of $S$. pneumoniae. from children with pneumonia in Shanghai. 19A/F, 6A/B, 23F and 14 were identified as the predominating serotypes in 2018. PCV13 serotype coverage was a little reduced than before. Compared with CAP, isolates from HAP had more PCV13/non-PCV7 serotypes, higher rate of PNSP and higher proportion of MDR. Moreover, our results revealed the type of clonal disseminations, and more attention should be paid to the emerging Sweden ${ }^{15 A}-25 /$ ST63 with the popularization of vaccines. lytA, ply, psaA, pavA, $s p x B, h \operatorname{tr} A$ and $\operatorname{clp} P$ were observed in all isolates, which may be candidates for next generation vaccines. Finally, long-term high-quality surveillance should be conducted to assess impact and effectiveness brought by vaccines, and provide a foundation for prevention strategies and vaccine policies.

\section{DATA AVAILABILITY}

All datasets generated for this study are included in the manuscript.

\section{AUTHOR CONTRIBUTIONS}

HZ, WZ, and FP conceived and designed the experiments. WZ and YYS performed antibiotic susceptibility testing and serotyping. WZ and BW performed MLST and virulence genes detection. HZ, YS, TZ, and CW contributed strains and case data collection. WZ wrote the first draft of the manuscript, and all authors contributed to manuscript revision, read and approved the submitted version.

\section{FUNDING}

This study was funded by the Shanghai Municipal Commission of Health and Family Planning (2015ZB0203).

\section{ACKNOWLEDGMENTS}

We thank all members of the Clinical Laboratory of Shanghai Children's Hospital for their cooperation and technical help. 


\section{REFERENCES}

Arushothy, R., Ahmad, N., Amran, F., Hashim, R., Samsudin, N., and Azih, C. R. C. (2019). Pneumococcal serotype distribution and antibiotic susceptibility in Malaysia: a four-year study (2014-2017) on invasive paediatric isolates. Int. J. Infect. Dis. 80, 129-133. doi: 10.1016/j.ijid.2018.12.009

Bradley, J. S., Byington, C. L., Shah, S. S., Alverson, B., Carter, E. R., Harrison, C., et al. (2011). The management of community-acquired pneumonia in infants and children older than 3 months of age: clinical practice guidelines by the Pediatric Infectious Diseases Society and the Infectious Diseases Society of America. Clin. Infect Dis. 53:e25-76. doi: 10.1093/cid/cir531

Brooks, L. R. K., and Mias, G. I. (2018). Streptococcus pneumoniae's virulence and host immunity: aging, diagnostics, and prevention. Front. Immunol. 9:1366. doi: 10.3389/fimmu.2018.01366

Bryant, J. C., Dabbs, R. C., Oswalt, K. L., Brown, L. R., Rosch, J. W., Seo, K. S., et al. (2016). Pyruvate oxidase of Streptococcus pneumoniae contributes to pneumolysin release. BMC Microbiol. 16:271. doi: 10.1186/s12866-016-0881-6

Cai, K., Wang, Y., Guo, Z., Xu, X., Li, H., and Zhang, Q. (2018). Clinical characteristics and antimicrobial resistance of pneumococcal isolates of pediatric invasive pneumococcal disease in China. Drug Resist. 11, 2461-2469. doi: 10.2147/IDR.S183916

Cawcutt, K., and Kalil, A. C. (2017). Pneumonia with bacterial and viral coinfection. Curr. Opin. Crit. Care. 23, 385-390. doi: 10.1097/MCC.0000000000000435

Chen, K., Zhang, X., Shan, W., Zhao, G., and Zhang, T. (2018). Serotype distribution of Streptococcus pneumoniae and potential impact of pneumococcal conjugate vaccines in China: a systematic review and meta-analysis. Hum. Vacc. Immunotherapeut. 14, 1453-1463. doi: 10.1080/21645515.2018.1435224

Coffey, T. J., Enright, M. C., Daniels, M., Morona, J. K., Morona, R., Hryniewicz, W., et al. (1998). Recombinational exchanges at the capsular polysaccharide biosynthetic locus lead to frequent serotype changes among natural isolates of Streptococcus pneumoniae. Mol. Microbiol. 27, 73-83. doi: 10.1046/j.1365-2958.1998.00658.x

Cornick, J. E., Tastan Bishop, O., Yalcin, F., Kiran, A. M., Kumwenda, B., Chaguza, C., et al. (2017). The global distribution and diversity of protein vaccine candidate antigens in the highly virulent Streptococcus pnuemoniae serotype 1. Vaccine. 35, 972-980. doi: 10.1016/j.vaccine.2016.12.037

Dalcin, D., Sieswerda, L., Dubois, S., and Ulanova, M. (2018). Epidemiology of invasive pneumococcal disease in indigenous and non-indigenous adults in northwestern Ontario, Canada, 2006-2015. BMC Infect. Dis. 18:621. doi: 10.1186/s12879-018-3531-9

Enright, M. C., and Spratt, B. G. (1998). A multilocus sequence typing scheme for Streptococcus pneumoniae: identification of clones associated with serious invasive disease. Microbiology 144 (Pt 11), 3049-3060. doi: 10.1099/00221287-144-11-3049

Falup-Pecurariu, O. (2012). Lessons learnt after the introduction of the seven valent-pneumococcal conjugate vaccine toward broader spectrum conjugate vaccines. Biomed. J. 35, 450-456. doi: 10.4103/2319-4170.104409

Feil, E. J., Li, B. C., Aanensen, D. M., Hanage, W. P., and Spratt, B. G. (2004). eBURST: inferring patterns of evolutionary descent among clusters of related bacterial genotypes from multilocus sequence typing data. J. Bacteriol. 186, 1518-1530. doi: 10.1128/JB.186.5.1518-1530.2004

Fu, J., Li, L., Liang, Z., Xu, S., Lin, N., Qin, P., et al. (2019). Etiology of acute otitis media and phenotypic-molecular characterization of Streptococcus pneumoniae isolated from children in Liuzhou, China. BMC Infect. Dis. 19:168. doi: $10.1186 /$ s12879-019-3795-8

GBD 2016 Lower Respiratory Infections (2018). Estimates of the global, regional, and national morbidity, mortality, and aetiologies of lower respiratory infections in 195 countries, 1990-2016: a systematic analysis for the Global Burden of Disease Study 2016. Lancet Infect. Dis. 18, 1191-1210. doi: 10.1016/S1473-3099(18)30310-4

Gentile, A., Bardach, A., Ciapponi, A., Garcia-Marti, S., Aruj, P., Glujovsky, D., et al. (2012). Epidemiology of community-acquired pneumonia in children of Latin America and the Caribbean: a systematic review and meta-analysis. Int. J. Infect. Dis. 16:e5-15. doi: 10.1016/j.ijid.2011.09.013

Hackel, M., Lascols, C., Bouchillon, S., Hilton, B., Morgenstern, D., and Purdy, J. (2013). Serotype prevalence and antibiotic resistance in Streptococcus pneumoniae clinical isolates among global populations. Vaccine. 31, 4881-4887. doi: 10.1016/j.vaccine.2013.07.054

Ibrahim, Y. M., Kerr, A. R., McCluskey, J., and Mitchell, T. J. (2004). Role of HtrA in the virulence and competence of Streptococcus pneumoniae. Infect. Immun. 72, 3584-3591. doi: 10.1128/IAI.72.6.3584-3591.2004

Janapatla, R. P., Chen, C. L., Hsu, M. H., Liao, W. T., and Chiu, C. H. (2018). Immunization with pneumococcal neuraminidases NanA, NanB and NanC to generate neutralizing antibodies and to increase survival in mice. J. Med. Microbiol. 67, 709-723. doi: 10.1099/jmm.0.000724

Jin, P., Xiao, M., Kong, F., Oftadeh, S., Zhou, F., Liu, C., et al. (2009). Simple, accurate, serotype-specific PCR assay to differentiate Streptococcus pneumoniae serotypes 6A, 6B, and 6C. J. Clin. Microbiol. 47, 2470-2474. doi: 10.1128/JCM.00484-09

Johnson, H. L., Deloria-Knoll, M., Levine, O. S., Stoszek, S. K., Freimanis Hance, L. Reithinger, R., et al. (2010). Systematic evaluation of serotypes causing invasive pneumococcal disease among children under five: the pneumococcal global serotype project. PLoS Med. 7:e1000348. doi: 10.1371/journal.pmed.1000348

Kang, L. H., Liu, M. J., Xu, W. C., Cui, J. J., Zhang, X. M., Wu, K. F., et al. (2016). Molecular epidemiology of pneumococcal isolates from children in China. Saudi Med. J. 37, 403-413. doi: 10.15537/smj.2016.4.14507

Keller, L. E., Robinson, D. A., and McDaniel, L. S. (2016). Nonencapsulated Streptococcus pneumoniae: emergence and pathogenesis. mBio. 7:e01792. doi: $10.1128 / \mathrm{mBio} .01792-15$

Li, L., Fu, J., Li, S., Guo, D., Chen, Z., Chen, S., et al. (2018). Phenotypic and molecular characterization of Streptococcus pneumoniae in pre-conjugate vaccine era: a Chinese hospital-based retrospective study. Vaccine. 36, 599-605. doi: 10.1016/.j.vaccine.2017.12.063

Linares, J., Ardanuy, C., Pallares, R., and Fenoll, A. (2010). Changes in antimicrobial resistance, serotypes and genotypes in Streptococcus pneumoniae over a 30-year period. Clin. Microb. Infect. 16, 402-410. doi: 10.1111/j.1469-0691.2010.03182.x

Maraki, S., Mantadakis, E., and Samonis, G. (2010). Serotype distribution and antimicrobial resistance of adult Streptococcus pneumoniae clinical isolates over the period 2001-2008 in Crete, Greece. Chemotherapy. 56, 325-332. doi: $10.1159 / 000320152$

Maraki, S., Mavromanolaki, V. E., Stafylaki, D., Hamilos, G., and Samonis, G. (2018). The evolving epidemiology of serotype distribution and antimicrobial resistance of streptococcus pneumoniae strains isolated from adults in crete, Greece, 2009-2016. Infect. Chemother. 50, 328-339. doi: $10.3947 /$ ic.2018.50.4.328

Miyazaki, H., Shibuya, R., Midorikawa, N., Chang, B., Ohnishi, M., and Matsumoto, T. (2017). Serotype distribution and antimicrobial susceptibility of Streptococcus pneumoniae strains isolated in Japan after introduction of the routine immunization program. J. Infect. Chemother. 23, 234-240. doi: 10.1016/j.jiac.2016.12.016

Naito, S., Tanaka, J., Nagashima, K., Chang, B., Hishiki, H., Takahashi, Y., et al. (2016). The impact of heptavalent pneumococcal conjugate vaccine on the incidence of childhood community-acquired pneumonia and bacteriologically confirmed pneumococcal pneumonia in Japan. Epidemiol. Infect. 144, 494-506. doi: $10.1017 /$ S0950268815001272

Nguyen, T. K., Tran, T. H., Roberts, C. L., Fox, G. J., Graham, S. M., and Marais, B. J. (2017). Risk factors for child pneumonia - focus on the Western Pacific Region. Paediatr. Respir. Rev. 21, 95-101. doi: 10.1016/j.prrv.2016.07.002

Pai, R., Gertz, R. E., and Beall, B. (2006). Sequential multiplex PCR approach for determining capsular serotypes of Streptococcus pneumoniae isolates. J. Clin. Microbiol. 44, 124-131. doi: 10.1128/JCM.44.1.124-131.2006

Pan, F., Han, L., Huang, W., Tang, J., Xiao, S., Wang, C., et al. (2015b). Serotype distribution, antimicrobial susceptibility, and molecular epidemiology of Streptococcus pneumoniae Isolated from Children in Shanghai, China. PLoS ONE. 10:e0142892. doi: 10.1371/journal.pone.0142892

Pan, F., Han, L., Kong, J., Wang, C., Qin, H., Xiao, S., et al. (2015a). Serotype distribution and antimicrobial resistance of Streptococcus pneumoniae causing noninvasive diseases in a Children's Hospital, Shanghai. Braz. J. Infect. Dis. 19, 141-145. doi: 10.1016/j.bjid.2014.08.010

Patel, K., and Goldman, J. L. (2016). Safety concerns surrounding quinolone use in children. J. Clin. Pharmacol. 56, 1060-1075. doi: 10.1002/jcph.715

Pneumococcal vaccines WHO position paper (2012). Releve Epidemiologique Hebdomadaire, Vol. 87 (Geneva), 129-44. 
Qin, L., Kida, Y., Imamura, Y., Kuwano, K., and Watanabe, H. (2013). Impaired capsular polysaccharide is relevant to enhanced biofilm formation and lower virulence in Streptococcus pneumoniae. J. Infect. Chemother. 19, 261-271. doi: 10.1007/s10156-012-0495-3

Ricketson, L. J., Wood, M. L., Vanderkooi, O. G., MacDonald, J. C., Martin, I. E., Demczuk, W. H., et al. (2014). Trends in asymptomatic nasopharyngeal colonization with Streptococcus pneumoniae after introduction of the 13-valent pneumococcal conjugate vaccine in Calgary, Canada. Pediatr. Infect. Dis. J. 33, 724-730. doi: 10.1097/INF.0000000000000267

Sader, H. S., Flamm, R. K., Streit, J. M., Carvalhaes, C. G., and Mendes, R. E. (2018). Antimicrobial activity of ceftaroline and comparator agents tested against organisms isolated from patients with community-acquired bacterial pneumonia in Europe, Asia, and Latin America. Int. J. Infect. Dis. 77, 82-86. doi: 10.1016/j.ijid.2018.10.004

Sader, H. S., Mendes, R. E., Le, J., Denys, G., Flamm, R. K., and Jones, R. N. (2019). Antimicrobial susceptibility of Streptococcus pneumoniae from North America, Europe, Latin America, and the Asia-Pacific Region: Results From 20 Years of the SENTRY Antimicrobial Surveillance Program (1997-2016). Open Forum Infect Dis. 6(Suppl 1):S14-s23. doi: 10.1093/ofid/ofy263

Shakrin, N. N., Masri, S. N., Taib, N. M., Nordin, S. A., Jamal, F., and Desa, M. N. (2014). Genotypic characterization of Malaysian human isolates of Streptococcus pneumoniae from carriage and clinical sources. Comp. Immunol. Microbiol. Infect. Dis. 37, 347-354. doi: 10.1016/j.cimid.2014.10.005

Shi, W., Li, J., Dong, F., Qian, S., Liu, G., Xu, B., et al. (2019). Serotype distribution, antibiotic resistance pattern, and multilocus sequence types of invasive Streptococcus pneumoniae isolates in two tertiary pediatric hospitals in Beijing prior to PCV13 availability. Expert Rev. Vacc. 18, 89-94. doi: 10.1080/14760584.2019.1557523

Shiri, T., Datta, S., Madan, J., Tsertsvadze, A., Royle, P., Keeling, M. J., et al. (2017). Indirect effects of childhood pneumococcal conjugate vaccination on invasive pneumococcal disease: a systematic review and meta-analysis. Lancet Glob. Health. 5:e51. doi: 10.1016/S2214-109X(16)30306-0

Smith, C. M., Sandrini, S., Datta, S., Freestone, P., Shafeeq, S., Radhakrishnan, P., et al. (2014). Respiratory syncytial virus increases the virulence of Streptococcus pneumoniae by binding to penicillin binding protein 1a. A new paradigm in respiratory infection. Am. J. Respir. Crit. Care Med. 190, 196-207. doi: 10.1164/rccm.201311-2110OC

Sonego, M., Pellegrin, M. C., Becker, G., and Lazzerini, M. (2015). Risk factors for mortality from acute lower respiratory infections (ALRI) in children under five years of age in low and middle-income countries: a systematic review and meta-analysis of observational studies. PLOS ONE. 10:e116380. doi: 10.1371/journal.pone.0116380
Steens, A., Bergsaker, M. A., Aaberge, I. S., Ronning, K., and Vestrheim, D. F. (2013). Prompt effect of replacing the 7 -valent pneumococcal conjugate vaccine with the 13-valent vaccine on the epidemiology of invasive pneumococcal disease in Norway. Vaccine. 31, 6232-6238. doi: 10.1016/j.vaccine.2013. 10.032

Suzuki, M., Dhoubhadel, B. G., Ishifuji, T., Yasunami, M., Yaegashi, M., Asoh, N., et al. (2017). Serotype-specific effectiveness of 23-valent pneumococcal polysaccharide vaccine against pneumococcal pneumonia in adults aged 65 years or older: a multicentre, prospective, test-negative design study. Lancet Infect. Dis. 17, 313-321. doi: 10.1016/S1473-3099(17)30049-X

Wahl, B., O'Brien, K. L., Greenbaum, A., Majumder, A., Liu, L., Chu, Y., et al. (2018). Burden of Streptococcus pneumoniae and Haemophilus influenzae type b disease in children in the era of conjugate vaccines: global, regional, and national estimates for 2000-15. Lancet Glob. Health. 6:e744-e57. doi: 10.1016/S2214-109X(18)30247-X

Weiser, J. N., Ferreira, D. M., and Paton, J. C. (2018). Streptococcus pneumoniae: transmission, colonization and invasion. Nat. Rev. Microbiol. 16, 355-367. doi: 10.1038/s41579-018-0001-8

Yan, Z., Cui, Y., Zhou, W., Li, W., Tan, X., Chen, W., et al. (2019). Molecular characterization of Streptococcus pneumoniae in children living in southwest China and assessment of a potential protein vaccine, rPfbA. Vaccine 37, 721-731. doi: 10.1016/j.vaccine.2018.12.021

Zhang, Q., Guo, Z., Bai, Z., and MacDonald, N. E. (2013). A 4 year prospective study to determine risk factors for severe community acquired pneumonia in children in southern China. Pediatr. Pulmonol. 48, 390-397. doi: 10.1002/ppul.22608

Zhao, C., Li, Z., Zhang, F., Zhang, X., Ji, P., Zeng, J., et al. (2017). Serotype distribution and antibiotic resistance of Streptococcus pneumoniae isolates from 17 Chinese cities from 2011 to 2016. BMC Infect. Dis. 17:804. doi: 10.1186/s12879-017-2880-0

Conflict of Interest Statement: The authors declare that the research was conducted in the absence of any commercial or financial relationships that could be construed as a potential conflict of interest.

Copyright (c) 2019 Zhao, Pan, Wang, Wang, Sun, Zhang, Shi and Zhang. This is an open-access article distributed under the terms of the Creative Commons Attribution License (CC BY). The use, distribution or reproduction in other forums is permitted, provided the original author(s) and the copyright owner(s) are credited and that the original publication in this journal is cited, in accordance with accepted academic practice. No use, distribution or reproduction is permitted which does not comply with these terms. 